\title{
High spatial resolution multiwavelength observations of star and planet formation
}

\author{
Mark J. McCaughrean \\ Astrophysikalisches Institut Potsdam, \\ An der Sternwarte 16, 14482 Potsdam, Germany
}

\begin{abstract}
We discuss the importance of multiwavelength (optical, infrared, millimetre, and radio), high angular resolution (arcsec to subarcsec) observations in studying star and planet formation. We provide a few illustrative examples, and briefly discuss how future observational facilities will allow a yet more detailed view of the important processes at work.
\end{abstract}

\section{Introduction}

The near environment of a young star is complex and dynamic. Gas and dust accretes from the parent molecular core via a circumstellar disk, where a planetary system may be forming. Simultaneously, highly-collimated bipolar jets and outflows drive some of that material away again. The star is likely part of a binary system, and/or in a dense cluster interacting with hundreds of other stars, perhaps including massive stars with intense ionising fluxes and winds.

The initial stages of cloud fragmentation and collapse can be studied with relatively low angular resolution single-dish millimetre telescopes, but once a protostar has formed with its attendant disk, jet, outflow, and nearby companions, higher angular resolution observations become vital. For example, the $60 \mathrm{AU}$ diameter of our own solar system (to Neptune) would subtend less than 0.5 arcsec at the nearest star-forming regions, the low-mass dark clouds (e.g., Taurus-Auriga at $\sim 140 \mathrm{pc}$ ), and less than 0.15 arcsec at regions of massive star formation in the Orion giant molecular clouds $(\sim 500 \mathrm{pc})$. In addition, there is a wide range in temperature $(\sim 10-10000 \mathrm{~K})$ and associated dust extinction $\left(\mathrm{A}_{V} \sim 0^{\mathrm{m}}-1000^{\mathrm{m}}\right)$ involved. Thus, to have a hope of disentangling the various formation and evolution processes at work, high angular resolution observations at optical, infrared, millimetre, and radio wavelengths are necessary.

Such observations are now routinely available and in this contribution, we shall present just a few (and as a consequence, mostly personally biased) recent case studies to illustrate the importance that (sub)arcsec angular resolution has in furthering our understanding of star and planet formation. We shall also briefly mention future advances in instrumentation that should allow us to make further significant breakthroughs in the coming decade. A more detailed review of recent star and planet formation observations carried out with large (8-10m class) optical/infrared telescopes, and of high-resolution imaging of very low-mass stars, brown dwarfs, and circumstellar disks in the Orion Trapezium Cluster can be found in McCaughrean $(2000,2001)$ respectively. 


\section{The jet-outflow connection: the case of $\mathbf{H H} 211$}

Young stars go through a phase where they eject bipolar outflows of cold molecular gas seen at millimetre wavelengths (Fukui etal. 1993) and in some cases, highly collimated jets of ionised and/or shocked molecular gas seen at optical/infrared wavelengths (Hartigan et al. 2000). One theoretical model holds that a magnetohydrodynamic $\mathrm{X}$-wind directly drives the broader outflow and also asymptotically converges to form a jet along the central axis (Shu et al. 2000). Another suggests that the jet is the prime mover, and that it entrains the outflow as it impacts the surrounding molecular medium (Raga \& Cabrit 1993). Problems in distinguishing between these two models arise in part due to the large difference in spatial resolution afforded by single-dish millimetre observations of the outflow ( $\gtrsim 10$ arcsec) and optical/infrared imaging of the jet $(\lesssim 1$ arcsec $)$. However, the advent of sensitive arcsec resolution millimetre interferometry has begun to clarify matters.

$\mathrm{HH} 211$ is a highly collimated jet associated with a very young Class 0 protostellar system near IC 348 in Perseus (McCaughrean, Rayner, \& Zinnecker 1994). The jet is seen only at infrared wavelengths, most notably in emission from shocked molecular hydrogen at $2.122 \mu \mathrm{m}$, and exhibits a peculiar bilateral symmetry about the axis of an associated cold molecular outflow. The jet is only $\sim 0.15 \mathrm{pc}$ long and thus probably only $\sim 10^{3} \mathrm{yrs}$ old, assuming typical jet velocities of $100-200 \mathrm{~km} \mathrm{~s}^{-1}$. As such, it is an ideal system in which to study the initial interaction of the jet with its surrounding medium.

Gueth \& Guilloteau (1999) were able to image HH 211 at arcsec resolution in the ${ }^{12} \mathrm{CO}(2-1)$ line using the Plateau de Bure millimetre interferometer (Figure 1). They found that the cold outflow is essentially coextensive with the shocked $\mathrm{H}_{2}$ of the jet, and that at low velocities, the $\mathrm{CO}$ emission is almost directly coincident with the $\mathrm{H}_{2}$ knots and thus bilaterally symmetric. At high velocities however, the $\mathrm{CO}$ is confined to a narrow jet down the centre of the $\mathrm{H}_{2}$ structure. This spatial and velocity structure can be well understood in terms of the Raga \& Cabrit (1993) model, in which the bowshock of the underlying highly collimated jet interacts with the ambient medium, and entrains cold ambient gas into an outflow. Thus at the very young age of $\mathrm{HH} 211$ at least, it appears as though the jet-driven outflow paradigm applies.

Further complementary optical/infrared and millimetre studies of jets and outflows as a function of age and luminosity are needed to extend this finding: sensitive wide-field, high-resolution optical/infrared imaging is available with the new 8m-class telescopes (e.g., VLT, Gemini, Subaru, Keck), while the millimetre and submillimetre observations will be possible with the SMA and ALMA.

\section{Extraction of angular momentum: the case of $\mathrm{HH} 212$}

As a perhaps inevitable side effect of star formation, jets and outflows can also play an important part in the process. Excess angular momentum must be shed from infalling molecular core material if any of it is to be accreted onto the central star. Viscous processes in the circumstellar disk may transport some angular momentum outwards, but a rotating jet and outflow may satisfy the same goal. 


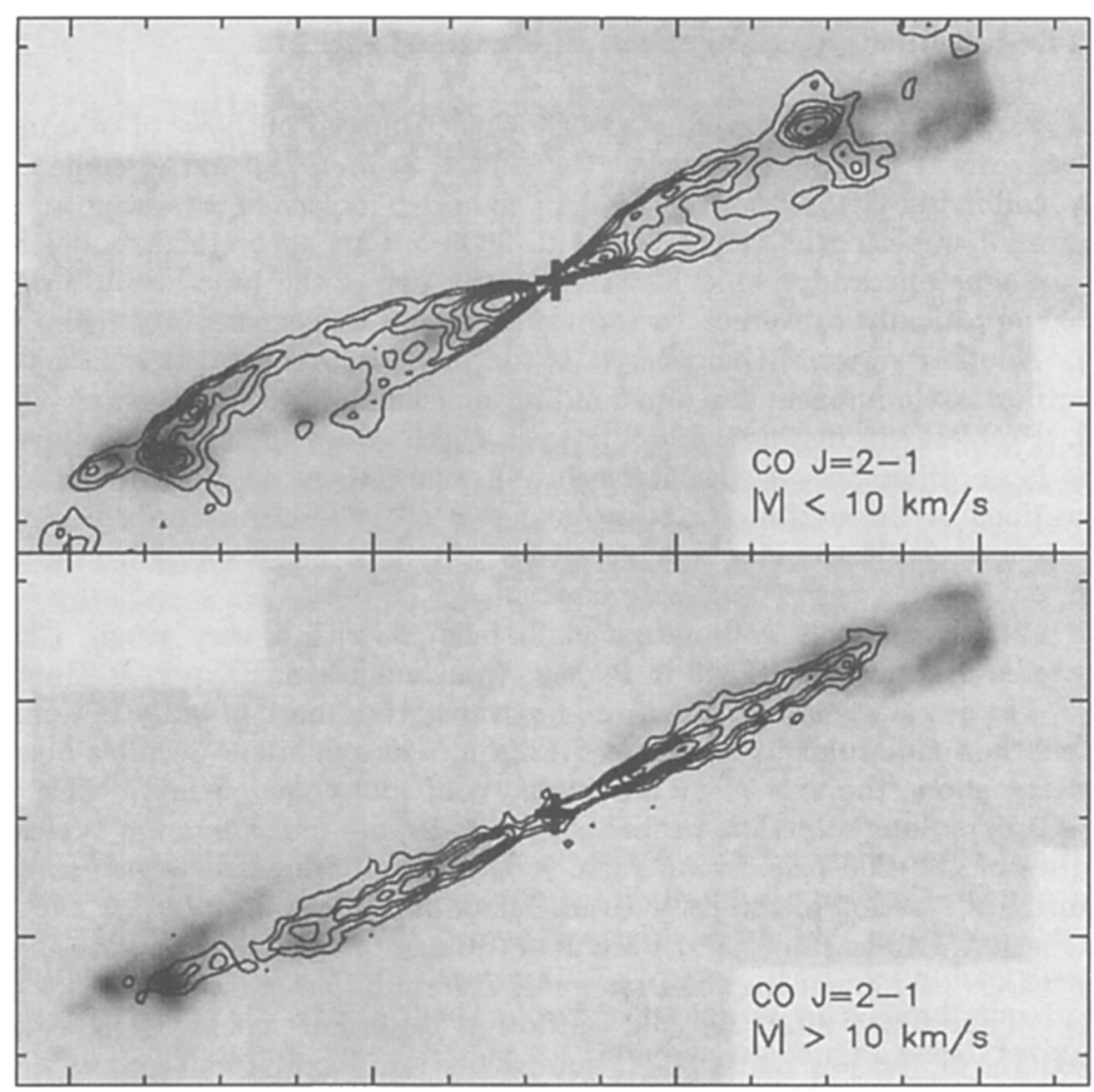

Figure 1. The HH 211 jet and outflow in Perseus. The jet is seen in grayscale in both panels in shocked molecular hydrogen at $2.122 \mu \mathrm{m}$ (McCaughrean, Rayner, \& Zinnecker 1994), exhibiting a peculiar bilateral symmetry. The contours show ${ }^{12} \mathrm{CO}(2-1)$ emission (Gueth \& Guilloteau 1999). The top panel shows low velocity CO emission largely coincident with the $\mathrm{H}_{2}$ structures, thus tracing the zone where the jet is interacting with the ambient medium and entraining it. The lower panel shows high velocity $\mathrm{CO}$ gas tracing out the true jet itself.

Until recently however, there has been almost no evidence for rotation in jets, so no angular momentum balance calculations have been possible.

$\mathrm{HH} 212$ is another very young jet seen only in shocked $\mathrm{H}_{2}$ emission at nearinfrared wavelengths, and is associated with a Class 0 protostar in Orion (Zinnecker, McCaughrean, \& Rayner 1998). The jet is highly symmetric, with a series of knots and bowshocks on one side that is almost exactly mirrored by an equivalent set on the other, the jet stretching over $0.4 \mathrm{pc}$ in total length (Figure 2). The implication of this symmetry is that the sequence of knots and bowshocks must be related to intrinsic variations at the star itself, a kind of ticker-tape record of its accretion history. Proper motion measurements in 


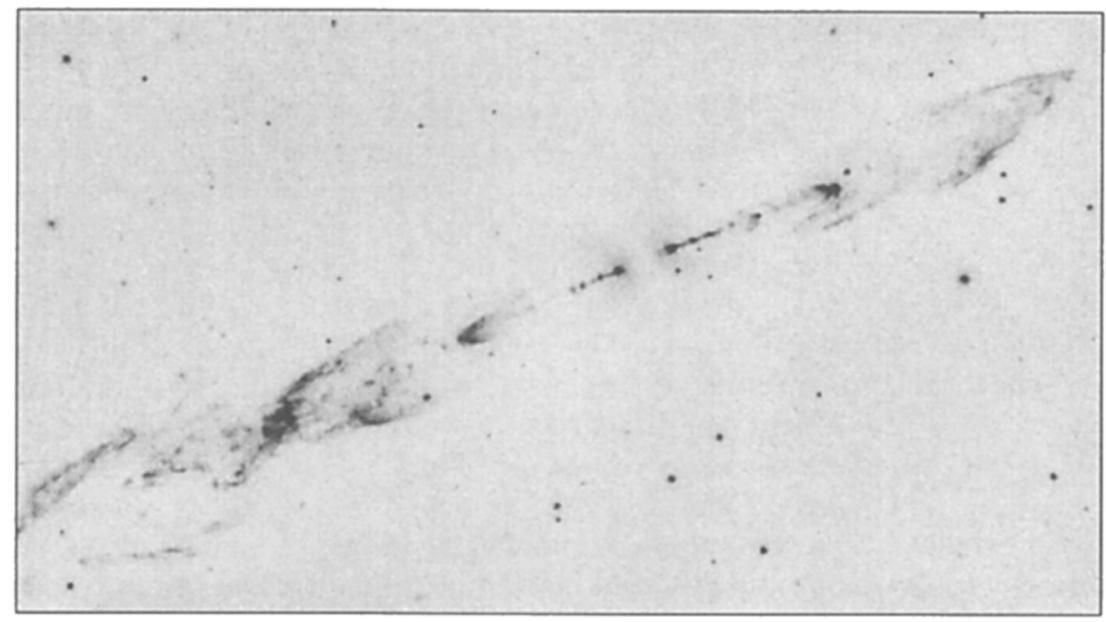

Figure 2. The HH212 jet seen in the $2.122 \mu \mathrm{m}$ line of molecular hydrogen. The jet exhibits a high degree of symmetry in the series of knots and bowshocks seen on either side of the embedded driving source. These data were taken with the infrared camera ISAAC on the $8.2 \mathrm{~m}$ VLT in October 2000. The integration time is 90 minutes for most of the jet, 45 minutes for the outer bowshocks; the resolution is seeinglimited at 0.35 arcsec FWHM (McCaughrean et al., in prep).

HH 212 show tangential velocities of $\sim 100-300 \mathrm{~km} \mathrm{~s}^{-1}$, both in the innermost knots as seen in $\mathrm{H}_{2}$ (McCaughrean etal., in prep), and at the base of the jet as seen in VLBA observations of $\mathrm{H}_{2} \mathrm{O}$ masers (Claussen et al. 1998). Small differences in radial velocities on the two sides of the jet show that it lies within a few degrees of the plane of the sky (Zinnecker et al. 1998; Claussen et al. 1998).

At high spatial resolution in the near-infrared, the innermost $\mathrm{H}_{2}$ knots are resolved into small bowshocks, with sizes typically 1 arcsec $(\sim 400 \mathrm{AU})$ transverse to the outflow direction. Davis et al. (2000) carried out velocity-resolved spectroscopy in the $2.122 \mu \mathrm{m}$ line to measure the radial velocities of the knots at three spatial positions transverse to the outflow axis. The spectra showed evidence for a systematic radial velocity gradient of $\sim 2.3 \mathrm{~km} \mathrm{~s}^{-1}$ over $\sim 1 \operatorname{arcsec}$ (400 AU) across some of the knots, which Davis et al. tentatively interpreted as being due to rotation in the jet.

At the same time, Wiseman et al. (2001) used the VLA in the $\mathrm{NH}_{3}(1,1)$ line to map the velocity structure of the large-scale (12000 AU FWHM diameter) flattened envelope from which the driving source of HH 212, IRAS 05413-0104, is probably still accreting. They found a smooth, constant $\sim 0.2 \mathrm{~km} \mathrm{~s}^{-1}$ radial velocity gradient over $\sim 22 \operatorname{arcsec}(8800 \mathrm{AU})$ transverse to the outflow axis, indicative of solid-body rotation at least in outer parts of the envelope: the Keplerian rotation that most probably exists further in may well be masked by optical depth and resolution effects.

The first important point is that the $\mathrm{H}_{2}$ and $\mathrm{NH}_{3}$ velocity gradients show that the jet and envelope rotate in the same sense. The second point is that the 
jet may indeed be able to carry away much of the envelope angular momentum. First we assume that the jet rotates at $1.15 \mathrm{~km} \mathrm{~s}^{-1}$ at a radius of $200 \mathrm{AU}$ (i.e., $2.3 \mathrm{~km} \mathrm{~s}^{-1}$ over $\left.400 \mathrm{AU}\right)$. Then the fraction of the disk mass that must be carried away by the jet in order to remove all of its angular momentum is given by the ratio of the disk velocity at $200 \mathrm{AU}$ radius to that of the jet. At one extreme, if we assume the apparent solid-body rotation in the $\mathrm{NH}_{3}$ core continues in to $200 \mathrm{AU}$, then the velocity there would be $0.005 \mathrm{~km} \mathrm{~s}^{-1}$. In this case, only $(0.005 / 1.15) \sim 0.5 \%$ of the disk material would have to be ejected in the jet. At the other extreme, assuming an underlying Keplerian rotation from the outer edge of the $\mathrm{NH}_{3}$ core inwards, the velocity at $200 \mathrm{AU}$ would be $\sim 0.47 \mathrm{~km} \mathrm{~s}^{-1}$. Then $(0.47 / 1.15) \sim 40 \%$ of the disk material would have to be in the jet.

Clearly these are very crude approximations, and the reality must be much more complicated. Nevertheless, HH 212 appears to provides at least broadly plausible evidence that the angular momentum problem may be solved by converting some fraction of the infalling material into a relatively rapidly rotating outflowing jet. Obviously however, these results are very preliminary, and substantially better rotation curves must be obtained both for infalling envelopes and disks, and their corresponding outflowing jets. The VLA and ALMA will provide the former data, while echelle spectrometers coupled with adaptive optics on large $8 \mathrm{~m}$-class telescopes will provide the latter.

\section{Direct imaging of circumstellar disks}

The present paradigm for star formation holds that planets form in dense disks of dust and gas. But despite ample evidence for such disks encircling young stars on one hand and for extrasolar planetary systems around nearby mature stars on the other, it is still not really known how these disks turn into planets, or at what efficiency, i.e., what fraction of all disks indeed develop planets. The fact that the discovery of massive planets in very close orbits around nearby stars was a surprise, indicates that this problem cannot be solved on the basis of theory alone; observations of the evolution of disks are needed.

Typical circumstellar disks have diameters of $\lesssim 500 \mathrm{AU}$, and subarcsecond resolution is required to make detailed probes of their structure in the nearest star-forming regions. It is now possible to resolve the cold gas and dust in nearby disks via millimetre interferometry (Wilner \& Lay 2000), but even higher resolution is available at optical/infrared wavelengths via the HST and adaptive optics. At these wavelengths, we are sensitive to scattering and absorption by dust (McCaughrean, Stapelfeldt, \& Close 2000).

A particular case in point are the disks seen around young stars in the Trapezium Cluster, in silhouette against the bright emission-line background of the Orion Nebula (O'Dell, Wen, \& Xu 1993; McCaughrean \& O'Dell 1996; Chen et al. 1998; McCaughrean et al. 1998; Bally, O'Dell, \& McCaughrean 2000). Figure 3 shows an HST montage of these disks, spanning a wide range of sizes and orientations. Several disks are seen edge-on, where if the central star is seen at all, it is via polar reflection nebulae, not directly, thus minimising contamination by direct stellar light.

Multiwavelength optical/infrared imaging of these edge-on disks can be used to probe their structure and dust properties at the outer edge. The disks are 


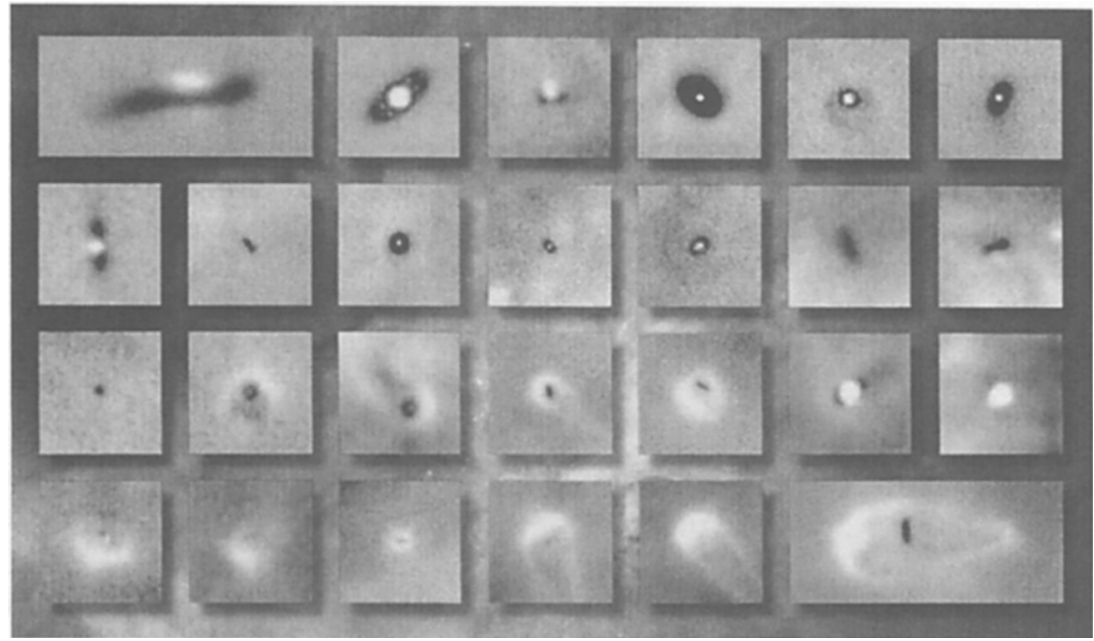

Figure 3. HST images of young silhouette disks in the Orion Nebula, associated with young $(0.5-2 \mathrm{Myr})$ low-mass $\left(0.3-1.5 \mathrm{M}_{\odot}\right)$ members of the Trapezium Cluster. Some of the disks are near edge-on and the central star cannot be seen directly; some others are embedded in ionised nebulae created as the disk is heated and ablated by the central OB stars in the cluster. Each panel is $1.67 \times 1.67$ arcsec or $750 \times 750 \mathrm{AU}$ in size (the two large panels are $1700 \times 750 \mathrm{AU}$ ); disk radii are $\sim 50$ $500 \mathrm{AU}$. The images were taken in the $\mathrm{H} \alpha$ filter of WFPC2 or the [O III] filter of STIS (from McCaughrean \& O'Dell 1996; Bally, O'Dell, \& McCaughrean 2000).

sharply truncated at some outer radius, but nevertheless have resolvable structure there (McCaughrean \& O'Dell 1996). Throop et al. (2001) have recently suggested that the apparent lack of change in transparency between 0.5 and $1.9 \mu \mathrm{m}$ at the edge of the large (900 AU diameter) edge-on disk Orion 114-426 is evidence for achromatic and thus large dust grains, with diameters of $5 \mu \mathrm{m}$ or greater, as compared to typical ISM grains at least ten times smaller. They present modelling results which suggest that if grain growth has proceeded to this degree at the outer edge, there should be significantly larger millimetreand even metre-sized rocks further in, where the disk is denser. In turn, this would suggest that planetesimals may form rather quickly and that planetary systems may already be developing in these $\sim 1$ Myr old disks. This result contrasts with that from HST imaging of similar edge-on disks seen around stars in Taurus-Auriga (e.g., HH 30: Burrows et al. 1996; HK Tau/c: Stapelfeldt et al. 1998), where little evidence for grain growth is seen.

In any case, the majority of the circumstellar disks in the Trapezium Cluster are being externally photoevaporated by the central OB stars, with the hot gas then forming a ionised bubble around the young parent star. The destruction timescale for these disks is thought to be rather short $\left(\sim 10^{5} \mathrm{yrs}\right.$; Hollenbach, Yorke, \& Johnstone 2000), and even if large grains have formed already, the disks may not survive long enough to form mature planetary systems. 
More detailed studies of the structure and evolution of disks in hazardous environments are needed, since the bulk of the galactic stellar population may form in such regions. Substantially higher resolution optical/infrared imaging will become available with ground-based adaptive optics (e.g., NAOS/CONICA on the VLT), space-based imaging (NGST), and, ultimately, interferometers (e.g., VLTI, LBT, Keck; cf. Malbet et al. 1999). A detailed examination of disk structure as a function of age and environment should yield important clues as to how disks may (or may not) form planetary systems.

\section{Young stellar clusters}

Thus far we have largely been discussing isolated stars, their jets, and their disks. However, a large fraction of the galactic field population is thought to form in dense, embedded clusters (Lada, Myers, \& Strom 1993), and thus a detailed assessment of their cumulative properties and the effects of such a crowded birth environment is vital if we are to understand the processes by which whole populations of stars form.

These clusters can have stellar densities of up to $10^{4}$ to $10^{5}$ stars per cubic parsec in their cores, yielding a typical projected linear separation of $\sim 1000 \mathrm{AU}$, or just 2 arcsec at $500 \mathrm{pc}$ and only 0.4 arcsec at a more typical distance of $3 \mathrm{kpc}$. In addition, there is often strong background emission from an $\mathrm{H}$ II region and significant associated dust. Thus high angular resolution infrared imaging is a prerequisite for separating individual stars, and for detecting faint, low-mass cluster stars and brown dwarfs close to brighter companions, against the nebular background, and through the extinction. As a concrete example, a $2 \mathrm{Myr}$ old $1 \mathrm{M}_{\text {Jup }}$ object with $A_{V} \sim 10^{\mathrm{m}}$ in a nearby star-forming regions at $150 \mathrm{pc}$ would have a $K$-band magnitude $\sim 20^{\mathrm{m}}$, and could be detected in a few hours with good subarcsec imaging on an $8 \mathrm{~m}$ class telescope.

Space constraints here preclude a detailed look at any recent results from young cluster studies. However, as a representative example, the importance of high angular resolution was highlighted in a VLT study of the distant (6$7 \mathrm{kpc}$ ) cluster NGC3603 by Brandl et al. (1999). Even at this distance, the large collecting area of the VLT and excellent seeing on Paranal ( $0.4 \operatorname{arcsec}$ FWHM) made it possible to resolve and detect stars down to $0.1 \mathrm{M}_{\odot}$, just above the hydrogen burning limit, in 45 minutes. Similarly deep, high angular resolution infrared imaging surveys have also been obtained of the Trapezium and RCW 38 clusters with the VLT (McCaughrean 2001; Alves et al., in prep). This will remain a very fertile area in the years to come, using the $8 \mathrm{~m}$ class telescopes for deep imaging surveys, followed up with multiobject classification spectroscopy (e.g., VIMOS/NIRMOS on the VLT), and even detailed proper-motion studies to ascertain cluster dynamics (e.g., PRIMA on the VLTI). The extraordinary sensitivity and wide-field diffraction-limited imaging capability of the NGST will extend these studies to very low mass limits right across the galaxy.

Acknowledgments. I would like to thanks Richard Schilizzi for inviting me to talk at this very interesting symposium, and for his patience in waiting for my written contribution. My work was funded in part by the EC Research Training Network on "The Formation and Evolution of Young Stellar Clusters" (RTN1-1999-00436) and DLR grant no. 50 OR 0004. 


\section{References}

Bally, J., O’Dell, C. R., \& McCaughrean, M. J. 2000, AJ, 119, 2919

Brandl, B., Brandner, W., Eisenhauer, F., Moffat, A. F. J., Palla, F., \& Zinnecker, H. 1999, A\&A, 352, L69

Burrows, C. J. et al. 1996, ApJ, 473, 437

Chen, H. et al. 1998, ApJ, 492, L173

Claussen, M. J., Marvel, K. B., Wootten, A., \& Wilking, B. A. 1998, ApJ, 507, L79

Davis, C. J., Berndsen, A., Smith, M. D., Chrysostomou, A., \& Hobson, J. 2000, MNRAS, 314, 241

Fukui, Y., Iwata, T., Mizuno, A., Bally, J., \& Lane, A. 1993, in E. H. Levy $\&$ J. I. Lunine, eds., Protostars \& Planets III, (Tucson: Univ. Arizona Press), p603

Gueth, F., \& Guilloteau, S. 1999, A\&A, 343, 571

Hartigan, P., Bally, J., Reipurth, B., \& Morse, J. A. 2000, in V. Mannings et al., eds., Protostars 8 Planets IV, (Tucson: Univ. Arizona Press), p841

Hollenbach, D. J., Yorke, H. W., \& Johnstone, D. 2000, in V. Mannings et al., eds., Protostars $\mathcal{E}$ Planets IV, (Tucson: Univ. Arizona Press), p401

Lada, E. A., Myers, P. C., \& Strom, K. M. 1993, in E. H. Levy \& J. I. Lunine, eds., Protostars \& Planets III, (Tucson: Univ. Arizona Press), p245

Malbet, F. et al. 1998, ApJ, 507, L149

McCaughrean, M. J. 2000, in J. Bergeron, ed., Discoveries and research prospects from 8-10 $m$ class telescopes, Proc. SPIE vol. 4005, 189

McCaughrean, M. J. 2001, in T. Montmerle \& P. André, eds., From Darkness to Light, ASP Conf. Ser., in press, 2001

McCaughrean, M. J. et al. 1998, ApJ, 492, L157

McCaughrean, M. J., \& O'Dell, C. R. 1996, AJ, 111, 1977

McCaughrean, M. J., Rayner, J. T., \& Zinnecker, H. 1994, ApJ, 436, L189

McCaughrean, M. J., Stapelfeldt, K. R., \& Close, L. M. 2000, in V. Mannings et al., eds., Protostars $\&$ Planets $I V$, (Tucson: Univ. Arizona Press), $\mathrm{p} 485$

O'Dell, C. R., Wen, Z., \& Hu, X. 1993, ApJ, 410, 696

Raga, A., \& Cabrit, S. 1993, A\&A, 278, 267

Shu, F. H., Najita, J., Shang, H., \& Li, Z.-Y. 2000, in V. Mannings et al., eds., Protostars \& Planets IV, (Tucson: Univ. Arizona Press), p789

Stapelfeldt, K. R., Krist, J. E., Ménard, F., Bouvier, J., Padgett, D. L., \& Burrows, C. J. 1998, ApJ, 502, L65

Throop, H. B., Bally, J., Esposito, L., \& McCaughrean, M. J. 2001, Science, submitted

Wilner, D. J., \& Lay, O. P. 2000, in V. Mannings et al., eds., Protostars \&6 Planets IV, (Tucson: Univ. Arizona Press), p509

Wiseman, J., Wootten, A., Zinnecker, H., \& McCaughrean, M. J. 2001, ApJ, in press (http://www.pha.jhu.edu/ jwiseman)

Zinnecker, H., McCaughrean, M. J., \& Rayner, J. T. 1998, Nature, 394, 862 